\title{
Estimation for torques applied to the master side in a construction robot teleoperation system
}

\author{
Lingtao Huang, Yanan Li, Tao Ni and Haidong Huang \\ Jilin University, School of Mechanical and Engineering, 130022 ChangChun, Jilin, China
}

\begin{abstract}
The purpose of this research is to develop a method that measures a torque which an operator applies to the joystick without using a torque/force sensor on a joystick. We dealt with a construction robot teleoperation system which is comprised of two joysticks as the master, and an excavator with four degrees of freedom consisting of fork glove, swing, boom, and arm as the slave. It is necessary to know the torque (force) exerted on the master side in force control. Because the joystick does not have a force/torque sensor in our lab, it is not possible to directly obtain the torque. To give prominence to the simple and practical construction robot teleoperation system in our lab, we proposed a method that measures the torque exerted on the joystick to existing equipment. Its effectiveness was verified by a reaction torque experiment.
\end{abstract}

\section{Introduction}

Teleoperation systems are employed to sense and operate objects at a distance. They are composed of a slave system and a master system. The slave side is composed of sensors to perceive remote environment, actuators to interact with it, and network channels to communicate with the master side [1]. In the master side, an operator commands the motion of the remote slave robot via a local master joystick [2]. The teleoperation system where the master side operator directly controls the remote robot according to information reflected from the remote environment is more effective and reliable [3].

We can have different classifications to joysticks which are applied to teleoperation systems according to different standards. The most common joysticks are movement-sensing joysticks because they respond to the tilt applied to the joystick's post [4]. On the other hand, isometric joysticks, whose post are rigid and do not deflect, respond to the forces applied to their transducers and theoretically require less strength and dexterity for transduction [5]. Since the mechanical abrasion is inevitable, the noncontact electronic joystick based on magnetic technology is much more precise and reliable than the mechanical joystick [6-8]. A lot of joysticks with 2-DOF [9] or 3-DOF [10] are implied in industrial applications. In teleoperation systems, there are a variety of feedback signals that flow from the remote environment to the master side [1], such as visual feedback [11] and force feedback [3].

In order to provide force feedback for the operator, an AC servo motor is used. And in order to measure the force which the operator applies to the joystick, a force sensor is attached to the rotational axis of each AC servo motor [12]. Recently, joysticks are commonly employed in many construction and industrial applications, such as cranes [13], excavators [14], and power wheelchairs [5]. It is necessary to know a torque (force) which an operator applies to a joystick in force control method. A common method is to install a force/torque sensor on the joystick.

In our lab, two joysticks, which are Microsoft Co., Ltd. manufactured and named as Side Winder Feedback 2, are introduced in our master-slave system. Because the joystick does not have a force/torque sensor, it is not possible to directly get the information of the torque applied to the joystick. To give prominence to the simple and practical construction robot teleoperation system in our lab, a method is proposed to measure the torque which an operator applies to the joystick to existing equipment in this research.

\section{Master side - joystick}

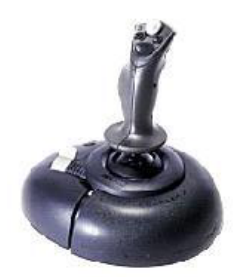

Figure 1. Joystick in a construction robot teleoperation system.

A typical teleoperation system consists of a master and a slave side. In our research, the master-slave system is composed of two joysticks, which act as the master, and a construction robot (a commercially available excavator 
modified based on Hitachi LandyKID-EX5) is the slave which has four links: fork glove, arm, boom and swing.

The joysticks enable movements forward/reverse and right/left, as in Figure 1. Its displacements are detected by position sensors. Each joystick is composed of two direct current motors that enable the operator to feel the sense of grasping an object by the fork glove and the work reaction torque (force sense) generated by the construction robot.

\section{Torque analysis and calculation}

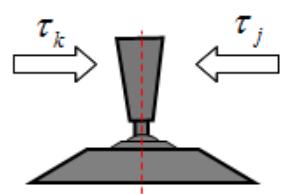

(a) Middle position

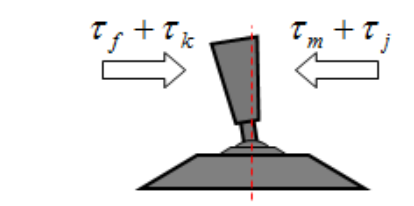

(b) Operation position without reaction torque

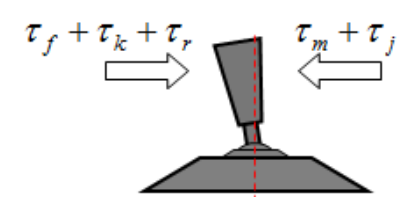

(c) Operation position with reaction torque

Figure 2. Joystick position and torques in horizontal placement.

Figure 2 shows the postures of the joystick and torques condition when the operator controls it in different operation position. In Figure 2, (a) shows the case that the joystick automatically places in middle position (zero position) when an operator does not manipulate it. In this case, a torque caused by handle's spring and damp effect $T_{k}$ overcomes a torque caused by handle's gravity $T_{j}$. (b) expresses the case that the joystick places in operation position without reaction torque generated by the construction robot ${ }_{r}{ }_{r}$. The operator applies an operation torque $T_{m}$ to the joystick, and $T_{f}$ is a torque caused by friction. (c) means that the joystick places in operation position with $\tau_{r}$, and there is a equilibrium equation for the torques $\tau_{f}+\tau_{k}++\tau_{r}=\tau_{m}+\tau_{j}$. In this research, a nondimensional quantity of torque 1 means $0.57 \mathrm{Nm}$.

To calculate the torque that the operator applied to the joystick, three problems should be examined and measured. First, the relationship between output torques measured by a torque meter and command torques to the joystick is examined. Second, the influence of handle's gravity is investigated and its mass is estimated. Third, $I_{f}$ is measured when the joystick is controlled and moved by the operator.

\subsection{Command torques and output torques}

To avoid the influence of handle's gravity, the joystick is placed vertically. Command torques is set to increase 5\% per 10 seconds. Output torques is measured by a torque meter. Torque meter is exerted on the center place of the joystick where the operator manipulates the handle. Experiment data is shown as Figure 3. The horizontal axis is the command torque, and vertical axis is the output torque. Due to the effect of friction, the output signal is zero though the command torques act on the joystick in range of \pm 0.1 . We can see that the relationship between the command signals and output signals is described as linear except ain the initial stage of input signals (near 0.1 ), and the fitting line is expressed by

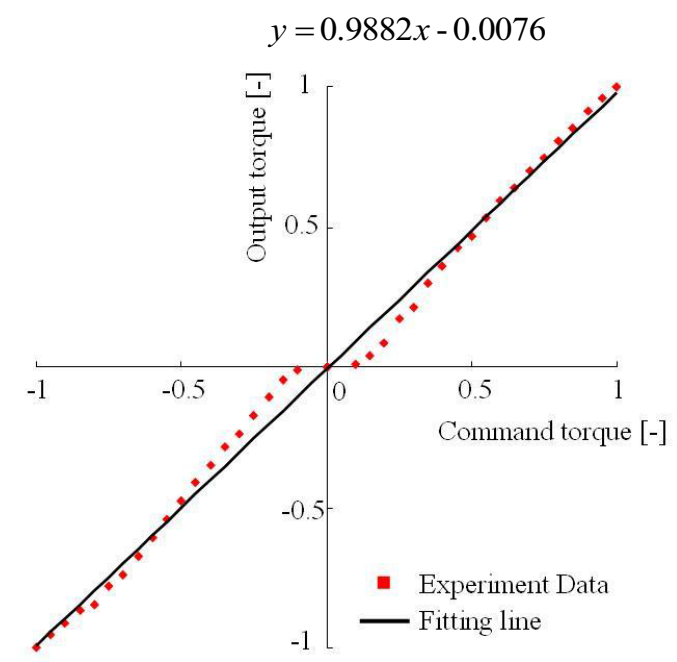

Figure 3. Command torques and output torques.

\subsection{Gravity Compensation}

As shown in Figure 2(b), there is the influence of handle's gravity when the joystick does not place in middle position. To calculate and measure the gravity of handle, experiment which measures the relationship between reaction torque to joystick and joystick displacement, is conducted when the joystick is placed horizontally and vertically, respectively.

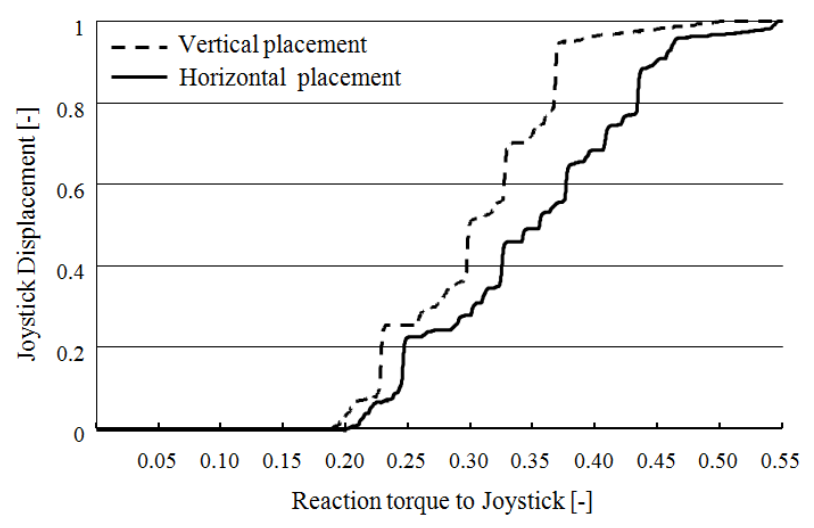

Figure 4. Reaction torque to joystick and joystick displacement.

When a control computer connected with the joystick send a command signal (reaction torque signal) to the joystick, the torque signal slowly and continuously increases $5 \%$ per $10 \mathrm{~s}$. The relationship between torque to the joystick and the joystick displacement is shown as Figure 4. The horizontal axis expresses the torque to joystick, and the vertical axis shows the joystick 
displacement, which is non-dimensional quantity and 1 denotes $0.06 \mathrm{~m}$. The dash line and the black line express the experiment data when the joystick was placed in vertical and horizontal placement, respectively. Because of the influence of the handle's gravity, a slightly significant discrepancy is observed between the red line and black line.

From Figure 2(b), it is known that the torque caused by handle's gravity can be expressed as follows:

$$
\tau_{j}=m g \times \sin (\theta) \times L
$$

Where $m$ is the mass of handle, and $L$ is the length between the center of rotation and a point that the operator applies a force to the handle. $\theta$ means the angle between the handle and vertical direction.

$\tau_{j}$ can be calculated in term of the data in Figure 4. It is known that there are two torques while the joystick places in a displacement, and the difference between the torques is considered as the action of $\tau_{j}$. So if angle $\theta$ is known, the handle's gravity can be calculated by fitting. Before calculating the gravity, it is necessary to find out the relationship between the joystick displacement and its angle.

\subsection{Relationship between joystick displacement and angle}

Experiment was conducted with the condition that the joystick was placed in vertical, and an angle senor was attached on the handle. Command torques are sent to the joystick and the joystick is moved slowly in experiment. The joystick displacement and angle are shown as Figure 5. The horizontal axis expresses the joystick displacement, and the vertical axis shows the angle of the handle, whose maximal absolute value is 16 degree. The red dots mean experiment data. In term of the experiment data, a fitting curve consists of three lines, which is shown as black lines and the mathematic expression of the curve is shown as follows:

$$
y=\left\{\begin{array}{cc}
19.7484 x+2.71992 & x \leq-0.34 \\
10.241 x-0.12867 & |\mathrm{x}|<0.34 \\
19.2728 x-3.09612 & x \geq 0.34
\end{array}\right.
$$

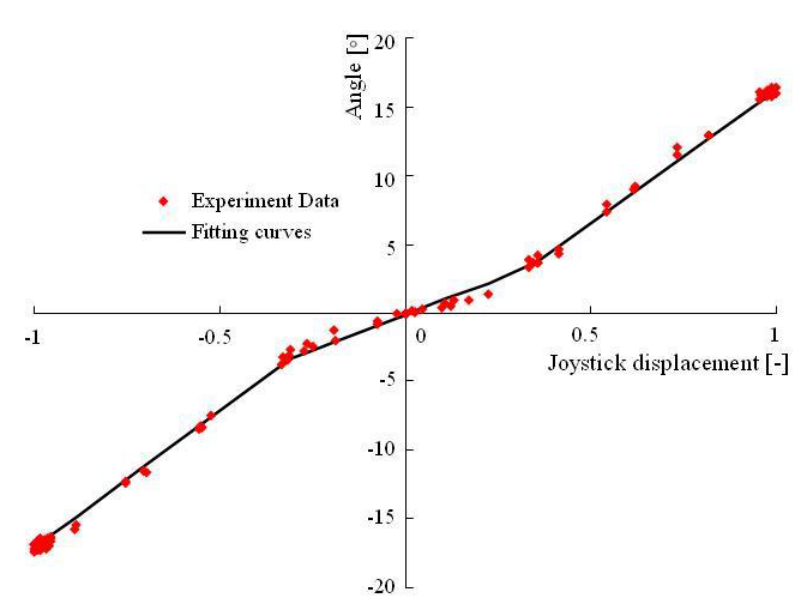

Figure 5. Joystick displacement and angles of the handle.

\subsection{Torque Caused by the gravity of handle}

Figure 6 shows the experiment data (black dots) and a fitting line, which are expressed as equation (4). The horizontal axis is the $\sin (\theta)$, and the vertical axis shows the torques. In term of the equation (1), since the parameters $(m g L)$ are constant values, there should be a proportional relationship between $\sin (\theta)$ and torque. Since the influence of friction and measurement error, it is considered that $0.0071 \mathrm{Nm}$ is a part of $\tau_{f}$, and then the value of $m g L$ is $0.1565 \mathrm{Nm}$.

$$
y=0.1565 x+0.0071
$$

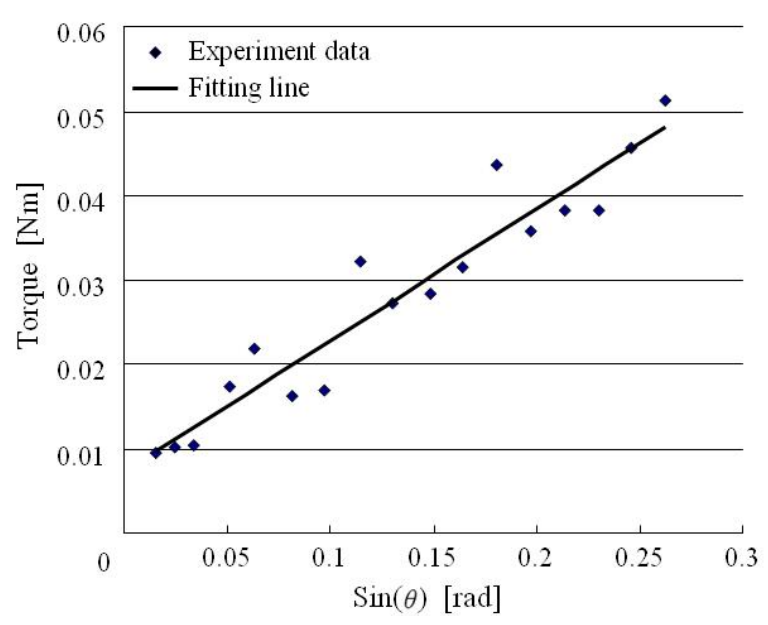

Figure 6. Estimation for $m g L$.

\subsection{Relationship between joystick displacement and torque to joystick}

A torque meter is used to slowly push the handle of the joystick to simulate the case that an operator grasps the joystick and moves it. Experiment is conducted under the joystick in horizontal placement on a table. Experiment data are collected when the joystick is moved from zero position to maximal position by the torque meter.

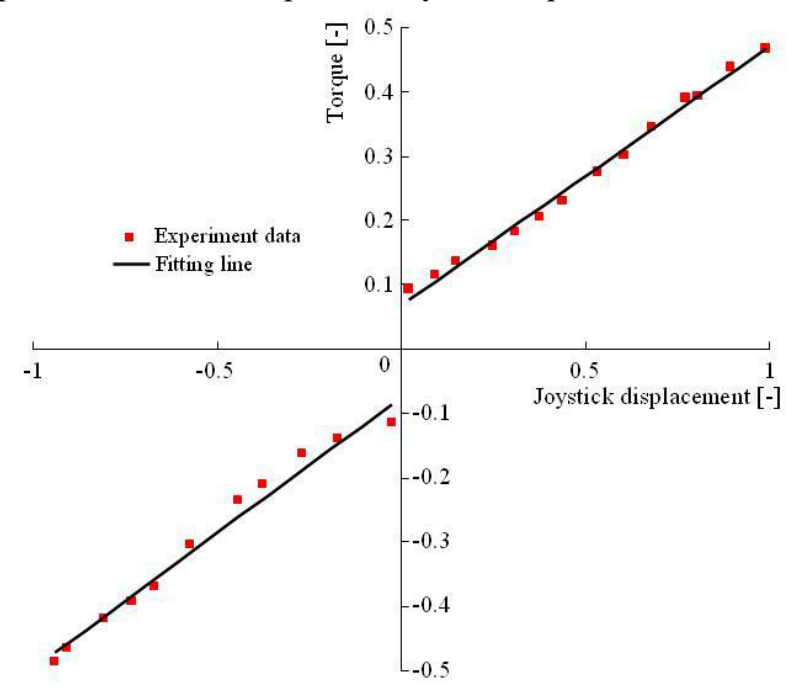

Figure 7. Relationship between torque to joystick and joystick displacement.

Red dots in Figure 7 show the data recorded by the torque meter, which are just like the torques applied to the 
joystick by an operator. $T_{j}$ can be calculated in term of equation (2), then the value $\tau_{f}+\tau_{k}$ equals to $\tau_{m}+\tau_{j}$ in term of Figure 2(b), which is shown as red dots in Figure 7. By fitting, equation (5) can be obtained and shown as follows:

$$
y=\left\{\begin{array}{ll}
0.4010 x+0.0681 & x>0 \\
0.4202 x-0.0757 & x<0
\end{array} .\right.
$$

In general, $\tau_{r}$ which is the reaction torque generated by the construction robot, is changed to an actual torque by equation (1). The torque $\tau_{j}$ caused by handle's gravity, is calculated by equation (2) and (3) in term of joystick displacement. The sum of $\tau_{f}$ and $\tau_{k}$ can be obtained by equation (5). Then torques exerted on the joystick by an operator $\tau_{m}$ is calculated by the equation $\tau_{f}+\tau_{k}++\tau_{r}=\tau_{m}+\tau_{j}$.

\section{Experiment}

Reaction torque experiment was conducted as follows: firstly, $\tau_{r}$ was set to a constant reaction torque -0.5 , the torque meter pushed the joystick from zero position to maximal position and collected experiment data, which are shown as "Fixed torque" in Figure 8. The red line is the result in term of equations (1), (2) and (5), blue dots are experiment data collected by torque meter. Secondly, $\tau_{r}$ was set to be increased from 0 to 1 and changed 5\% per $10 \mathrm{~s}$, and the joystick was pushed to 0.528391 position by the torque meter. Experiment data are "Fixed position" in Figure 8 , the black line is the fitting result according to equations (1), (2) and (5), pink dots are the data collected by the torque meter. From two ground data, we can see that the fitting result almost equals to the measured result. It proofs that it is effective to calculate torques applied to the joystick by our proposed method.

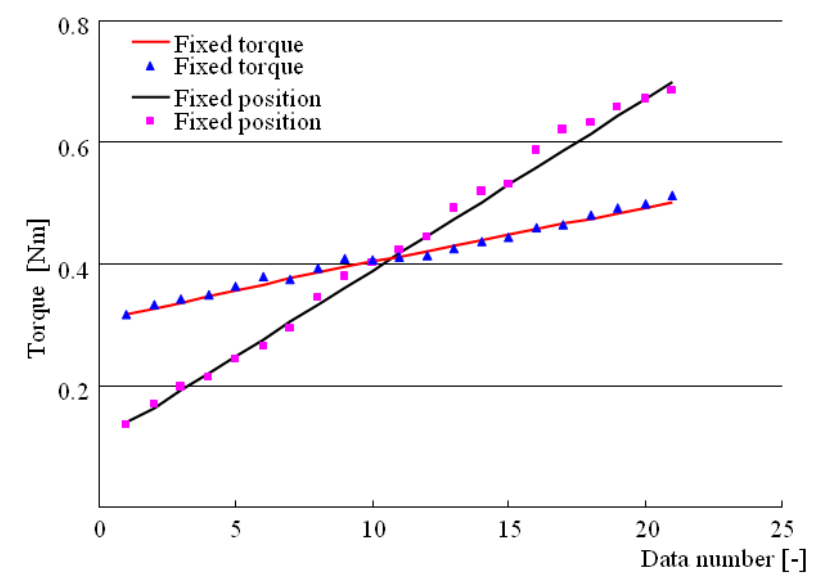

Figure 8. Reaction torque experiment.

\section{Conclusion}

The torque applied to a joystick by an operator must be known, when force control is introduced in a construction robot teleoperation system. In this research, a method was proposed by the authors, which measures a torque which an operator applies to the joystick without using a torque/force sensor on the joystick. In this research, we firstly examined the relationship between command torques controlled by a computer and output torques measured by a torque meter, then estimated the gravity of the handle by fitting, next, investigated and calculated relationship between $T_{k}$ and joystick displacement. Finally, reaction torque experiment proved the feasibility and validity of using the proposed control method in the construction robot teleoperation system.

\section{Acknowledgment}

The authors would like to thank the financial support from the National Natural Science Foundation of China (nos. 51575219 and nos. 51305153), Key Scientific Research Projects of Jilin Province (nos. 20130206034GX).

\section{References}

1. C. Pacchierotti, L. Meli, F. Chinello, M. Malvezzi, D. Prattichizzo. Int J Robot Res. 34, 1773-1787 (2015)

2. G. Niemeyer, J. J. E. Slotine. Int J Robot Res. 23, 873-890 (2004)

3. S. K. Cho, H. Z. Jin, J. M. Lee, B. Yao. Ieee-Asme T Mech. 15, 17-26 (2010)

4. B. E. Dicianno, H. Mahajan, R. A. Cooper. Pm\&R. 7, 555-561 (2015)

5. H. Mahajan, D. M. Spaeth, B. E. Dicianno, D. M. Collins, M. L. Boninger, R. A. Cooper. Arch Phys Med Rehab. 92, 1298-1304 (2011)

6. H. Jin, H. Lu, S. Cho, J. Lee. IEEE-ASME T Mech. 12, 549-556 (2007)

7. M. Kayal, F. Burger, R. S. Popovic. IEEE Sens J. 4, 759-763 (2004)

8. R. A. Cooper, D. K. Jones, S. Fitzgerald, M. L. Boninger, S. J. Albright. IEEE T Bio-Med Eng. 47, 902-910 (2000)

9. W. H. Li, B. Liu, P. B. Kosasih, X. Z. Zhang. Sensor Actuat a-Phys. 137, 308-320 (2007)

10. H. Zangl, T. Bretterklieber, G. Steiner, K. Riedmuller. 2007 IEEE Sensor. 1069-1072 (Atlanta, GA, 2007)

11. L. Zhang, Q. Huang, Y. P. Lu, T. Xiao,J. P. Yang, M. Keerio. 2006 IEEE/RSJ International Conference on Intelligent Robots and Systems. 1110-1115 (Beijing, CHINA, 2006)

12. A. Takemoto, K. Yano, T. Miyoshi, K. Terashima. 13th IEEE International Workshop on Robot and Human Interactive Communication. 533-538 (Kurashiki, JAPAN, 2004)

13. M. A. Huysmans, M. P. De Looze, M. J. M. Hoozemans, A. J. Van der Beek, J. H. Van Dieen. Ergonomics. 49, 1021-1035 (2006)

14. Y. J. Nam, M. K. Park. J Mech Sci Technol. 29, 397407 (2015) 